\title{
BLAP-tagged channel technology: a new direction trafficking toward epithelial physiology
}

\author{
Kirk L. Hamilton* \\ Department of Physiology, Otago School of Medical Sciences, University of Otago, Dunedin, New Zealand \\ ${ }^{*}$ Correspondence: kirk.hamilton@otago.ac.nz
}

\section{A commentary on}

Immunofluorescence-based assay to identify modulators of the number of plasma membrane KCa3.1 channels.

by Balut, C. M., Gao, Y., Luke, C., and Devor, D. C. (2010). Future Med. Chem. 2, 707-713.

Recent developments in fluorescent-probe technology have allowed real-time imaging of cellular events by engineering small peptide sequences [e.g., 15 amino acid sequence for a biotin-ligase acceptor peptide (BLAP), see Chen et al., 2005; Howarth and Ting, 2008] in an external domain of a protein (e.g., ion channel). The BLAP-tagged channels are biotinylated at the membrane with recombinant BirA (a biotin ligase from Escherichia coli) and labeled with a streptavidin-fluorophore, after which, the membrane and cellular fates of the BLAPtagged channels can be determined. The labeling of the BLAP-tagged channels is relatively rapid (less than $50 \mathrm{~min}$ ), very efficient (covalent binding), sensitive, and effective for protein detection by immunofluorescence and Western blot (Chen et al., 2005; Howarth and Ting, 2008).

Recently, Devor and colleagues (Balut et al., 2010b; Gao et al., 2010), using the BLAP-technology based on work of Ting, have defined the membrane residence and retrograde trafficking of the $\mathrm{Ca}^{2+}$-activated $\mathrm{K}^{+}$channels, $\mathrm{KCa} 3.1$ and $\mathrm{KCa} 2.3$, in human embryonic kidney (HEK293) cells and endothelial cells (HMEC-1). The engineering of the BLAP-tag within both $\mathrm{KCa} 3.1$ and $\mathrm{KCa} 2.3$ did not alter gating or $\mathrm{Ca}^{2+}$ dependence of the channels (Gao et al., 2010). Further, Gao et al. (2010) demonstrated that $\mathrm{KCa} 2.3$ had a long membrane residence time $(\sim 13 \mathrm{~h})$ and that the channel was recycled back to the membrane, while KCa3.1 had a short membrane residence time ( $90 \mathrm{~min})$, and was destined for the lysosomes (Balut et al., 2010b).
Both KCa3.1 and $\mathrm{KCa} 2.3$ have been suggested as therapeutic targets in many diseases including endothelial dysfunction and hypertension (Kohler et al., 2010). Pharmacological modulation and/or altering membrane channel residence time of these channels have promise as potential therapeutic targets for ameliorating diseases (Kohler et al., 2010). The question is how does one quickly screen potential modulators of residence time and endocytosis of a channel in a membrane?

The focus of this Commentary is to highlight recent work of Devor and colleagues (Balut et al., 2010a) in which they developed a novel immunofluorescentbased assay to assess modulators of endocytosis of proteins (e.g., KCa3.1). With this technique, they were able to quickly determine the channel membrane residence time and rate of endocytosis of KCa.1. This establishes a crucial paradigm for the further investigation of this channel, and other membrane proteins, as a therapeutic target(s) for clinical use.

Initially, Devor and colleagues realized the time of labeling of KCa3.1 must be reduced in order to rapidly assess modulators of channel endocytosis, and for the effectiveness of their procedure to be functionally viable in a 96-well plate format assay approach with future application in a highthroughput scenario. Indeed, they reduced the time of labeling KCa3.1 from 50 min to less than $15 \mathrm{~min}$. This was accomplished by subcloning BLAP-tagged KCa3.1 (KCa3.1BLAP) and BirA [with a KDEL endoplasmic reticulum (ER) retention motif] into a bicistronic plasmid. Following this, a stable HEK293 cell line was established expressing both the BLAP-tagged KCa3.1 and BirAKDEL.So, as each channel subunit is synthesized it is also biotinylated, providing a high efficiency of biotinylation. This molecular engineering innovation greatly reduced the time of labeling and the increased efficiency of biotinylation the channel.
Devor and colleagues (Balut et al., 2010a) have provided exciting experimental data, and "proof of concept" of an immunofluorescence-based assay in which they screened a modulator of the endocytosis of KCa3.1-BLAP. The overall experimental paradigm was to label membrane surface KCa3.1-BLAP and total plasma membrane with different fluorophores to determine the intracellular/membrane location of the channel and determine the effects of inhibition of the endocytotic pathway on the membrane residence time of KCa3.1BLAP. The evidence for endocytosis of KCa3.1-BLAP was assessed by the absence of membrane colocalization of the fluorescent signals (i.e., endocytosis).

Briefly, experiments were conducted in a 96-well plate format in which, surface channels and membranes of control cells were labeled at $4^{\circ} \mathrm{C}$ to prevent endocytosis of the channel during the labeling procedure. Additionally, for experimental cells, only surface channels were labeled $\left(4^{\circ} \mathrm{C}\right)$ and were placed at $37^{\circ} \mathrm{C}$ for assessing channel membrane residence time and endocytosis. After $90 \mathrm{~min}$, experimental cells, prelabeled KCa3.1-BLAP, were then labeled at $4^{\circ} \mathrm{C}$ with a different fluorophore specific for the membrane and colocalization was assessed. This was an adaptation required for the use of an automated analysis system and to assure that only plasma membrane was labeled prior to fixation and permeabilization of the cells (Balut et al., 2010a). Balut et al. (2010a) clearly demonstrated that at time $0, \mathrm{KCa} 3$-BLAP was only present at the membrane with robust colocalization of fluorescent labeling and that channels were internalized (i.e., within vesicles) within $90 \mathrm{~min}$.

In order to assess endocytosis of $\mathrm{KCa} 3.1$ BLAP, Devor and colleagues incubated cells expressing KCa3.1-BLAP with UBEI-41, an inhibitor of ubiquitin-activating enzyme E; which blocks the first step of the ubiquitination pathway. Therefore, at time 0 , control 
cells were either labeled as described above, or only surface channels of experimental cells were labeled and placed at $37^{\circ} \mathrm{C}$ for $90 \mathrm{~min}$ and incubated in the presence or absence of the UBEI-41. Again, after $90 \mathrm{~min}$, membrane proteins were labeled at $4^{\circ} \mathrm{C}$. In the presence UBEI-41, KCa3.1-BLAP was retained at the membrane with similar colocalization of fluorescent signal as compared to control cells (i.e., only surface channels). Here, Devor and colleagues demonstrated they could capture a "snapshot" of trafficking of KCa3.1-BLAP and identify an inhibitor of endocytosis of KCa3.1-BLAP in a 96-well plate format. For experimental and analysis details, the reader is directed to Balut et al. (2010a); which will be freely available in April 2011.

The immunofluorescence assay described by Devor and colleagues has many desirable aspects that will contribute to advances in the trafficking physiology of channels. First, with this assay, the time for labeling of the channel is greatly reduced from $50 \mathrm{~min}$ down to less than $15 \mathrm{~min}$. This has further implications of saving time and cost of materials when screening hundreds of wells for a single experiment. Secondly, their experimental approach achieved high efficiency of biotinylation as the ER-retained biotin ligase biotinylates each channel subunit as it is synthesized. Finally, the immunofluorescence assay provides "proof of concept" that this paradigm can be used in high-throughput screening strategy for identifying potential small-molecule modulators of endocytosis of specific proteins (e.g., channels) of the plasma membrane.

Currently, Devor and colleagues (personal communication) are modifying the experimental design of the 96-well plate format immunofluorescence assay to examine the potential modulators of $\mathrm{KCa} 2.3$. Additionally, Devor is developing an assay, using KCa3.1BLAP technology, to examine the trafficking of the channel from the ER to the plasma membrane. This assay will certainly occupy a unique niche for advancing our understanding of anterograde trafficking of ion channels (and other proteins), and prove useful in determining the fate of membrane proteins targeted specifically to the apical or basolateral membrane of polarized epithelia.

\section{WHY IS THIS PAPER IMPORTANT FOR EPITHELIAL PHYSIOLOGISTS?}

We need to understand the basic trafficking of epithelial ion channels to determine the possible role(s) of ion channels in diseases. The novel immunofluorescent-tagging approach that Devor and colleagues have developed will undoubtedly foster an exciting research avenue for epithelial physiology. Devor and colleagues have clearly provided an assay that can identify "small-molecule modulators" of endocytosis that lead to increased numbers of channels at the membrane, and thus, can enhance a physiological response (Balut et al., 2010a). Certainly, we must keep our "research eyes" open for novel technologies that can be widely applied not just by epithelial physiologists, but also by many molecular cell physiologists to further our understanding of ion channel physiology. Exciting possibilities exist for those willing to take the "BLAP-tag leap" into a new technology.

\section{ACKNOWLEDGMENTS}

I thank Drs Chris P. Bolter and Marcelo Carattino for critically reading this manuscript and for many helpful comments.
I thank Drs Daniel C. Devor and Corina M. Balut for further clarification of experimental details that were not included in their paper (Balut et al., 2010a). This work was supported by a NZ Lottery Board grant and the Department of Physiology, University of Otago.

\section{REFERENCES}

Balut, C. M., Gao, Y., Luke, C., and Devor, D. C. (2010a). Immunofluorescence-based assay to identify modulators of the number of plasma membrane KCa3.1 channels. Future Med. Chem. 2, 707-713.

Balut, C. M, Gao, Y., Murray, S. A., Thibodeau, P. H., and Devor, D. C. (2010b). ESCRT-dependent targeting of plasma membrane localized $\mathrm{KCa} 3.1$ to the lysosomes. Am. J. Physiol., Cell Physiol. 299, C1015-C1027.

Chen, I., Howarth, M., Lin, Y., and Ting, A. Y. (2005). Site-specific labeling of cell surface proteins with biophysical probes using biotin ligase. Nat. Methods 2, 99-104.

Gao, Y., Balut, C. M., Bailey, M. A., Patino-Lopez, G., Shaw, S., and Devor, D. C. (2010). Recycling of the $\mathrm{Ca}^{2+}$-activated $\mathrm{K}^{+}$channel, $\mathrm{KCa} 2.3$, is dependent upon RME1, Rab35/EPI64C, and an N-terminal domain. J. Biol. Chem. 285, 17938-17953.

Howarth, M., and Ting, A. Y. (2008). Imaging proteins in live mammalian cells with biotin ligase and monovalent streptavidin. Nat. Protoc. 3, 534-545.

Kohler, R., Kaistha, B. P., and Wulff, H. (2010). Vascular $\mathrm{K}_{\mathrm{Ca}}$-channels as therapeutic targets in hypertension and restenosis disease. Expert Opin. Ther. Targets 14, 143-155.

Received: 20 October 2010; accepted: 29 November 2010; published online: 13 December 2010.

Citation: Hamilton KL (2010) BLAP-tagged channel technology: a new direction trafficking toward epithelial physiology. Front. Physio. 1:162. doi: 10.3389/fphys.2010.00162 This article was submitted to Frontiers in Renal and Epithelial Physiology, a specialty of Frontiers in Physiology. Copyright (c) 2010 Hamilton. This is an open-access article subject to an exclusive license agreement between the authors and the Frontiers Research Foundation, which permits unrestricted use, distribution, and reproduction in any medium, provided the original authors and source are credited. 